\title{
Propagation characteristics of periodic structures possessing twist and polar glide symmetries
}

\author{
Oskar Dahlberg, ${ }^{1, *}$, Fatemeh Ghasemifard ${ }^{1}$, Guido Valerio ${ }^{2}$, and Oscar Quevedo-Teruel ${ }^{1}$ \\ ${ }^{1}$ Department of Electromagnetic Engineering, KTH Royal Institute of Technology, 10044 Stockholm, Sweden \\ ${ }^{2}$ Laboratoire d'Électronique et Électromagnétisme, Sorbonne Université, 75005 Paris, France
}

Received: 21 November 2018 / Accepted: 4 March 2019

\begin{abstract}
In this article, we provide an overview of the current state of the research in the area of twist symmetry. This symmetry is obtained by introducing multiple periods into the unit cell of a periodic structure through a rotation of consecutive periodic deformations around a symmetry axis. Attractive properties such as significantly reduced frequency dispersion and increased optical density, compared to purely periodic structures, are observed. The direct link between the symmetry order and these properties is illustrated through numerical simulations. Moreover, polar glide symmetry is introduced, and is shown to provide even further control of the dispersion properties of periodic structures, especially when combined with twist symmetry. Twist symmetries can, with benefit, be employed in the development of devices for future communication networks and space applications, where fully metallic structures with accurate control of the dispersion properties are desired.
\end{abstract}

Keywords: higher symmetry / twist symmetry / polar glide symmetry / broken symmetry

\section{Introduction}

A periodic structure possesses a higher symmetry if its unit cell is invariant under the composition of a translation and another geometrical operation [1-4]. The unit cell of a higher-symmetric periodic structure is composed of $m$ subunit cells, where $m$ is the degree of symmetry to which the compound symmetry operation, $\mathcal{S}$, is applied. Therefore, a higher-symmetric structure with periodicity $p$ possesses both the conventional translational symmetry, $\mathcal{T}_{p}$, and the higher symmetry, $\mathcal{S}$. The generalized Floquet theorem presented by Oliner [4] proves that the Bloch modes in higher-symmetric structures are eigenmodes to both the translation operation, $\mathcal{T}_{p}$, and the compound operation, $\mathcal{S}$. The two operators are linked through the relationship $\mathcal{S}^{m}=\mathcal{T}_{p}$. From this relationship, it is deduced that the dispersion diagram for the full unit cell consists of $m$ subsets: the spectrum of the compound operator, $\mathcal{S}$, and its $m-1$ space harmonic branches [5]. The presence of such harmonics in the Brillouin diagram for the full unit cell results in a closing of band gaps for the first $m-1$ edge points.

Twist symmetry is one type of higher symmetry, where the additional geometrical operation is a rotation around a twist axis, called here a "twist operation" [6-9]. More specifically, a structure possesses an $m$-fold twist symmetry if

\footnotetext{
* Corresponding author: oskdah@kth.se
}

the compound geometrical operation consists of a translation of $p / m$ along a given axis (the periodicity axis) and a rotation of $2 \pi / m$ around the same axis, where $p$ is the periodicity of the unit cell, and $m$ is an integer number. Purely periodic structures, where the periodic operation simply consists of a translation of the unit cell, can also be named 1-fold twist.

The concept of polar glide symmetry was introduced in [6], where a structure is said to possess polar glide symmetry if its flat approximation of the structure possesses Cartesian glide symmetry, i.e., the additional geometric operation is a mirroring in a plane. However, it has been noted that this definition can be improved upon, and in [7], the true definition of polar glide symmetry is proposed. Here, the authors state that a structure possesses a perfect polar glide symmetry if the sub-unit cell is translated along a symmetry axis, followed by a mirroring with respect to a cylindrical surface, coaxial with the symmetry axis $[7,8]$.

The electromagnetic properties of structures possessing higher symmetries, and in particular twist and glide symmetries, were extensively studied in the $60 \mathrm{~s}$ and $70 \mathrm{~s}$ [1-4]. These initial works focused on the study of onedimensional structures, and the results have been applied in the design of forward and backward scanning leaky-wave antennas [10-12]. More recently, glide symmetry has received renewed attention and it has been observed that glide-symmetric structures are optically denser and less dispersive than their purely periodic counterparts [13-16]. Moreover, purely periodic structures (i.e. non-glide) 


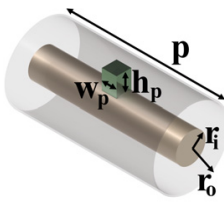

(a)

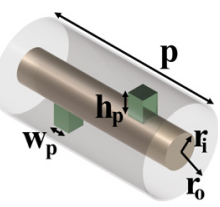

(b)

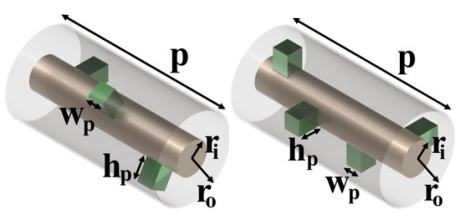

(c)

(d)

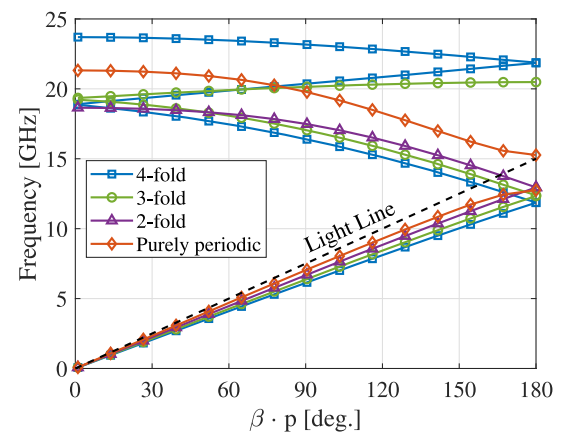

(e)

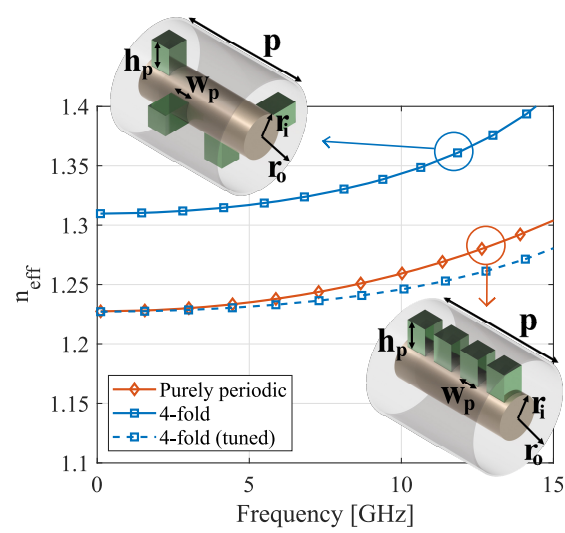

(f)

Fig. 1. Simulated perfect twist-symmetric structures with pin-type deformations. Studied structures: (a) purely periodic, (b) 2-fold, (c) 3-fold, and (d) 4-fold. (e) Dispersion diagram using the dimensions: $h_{p}=1.2 \mathrm{~mm}, w_{p}=1 \mathrm{~mm}, r_{i}=1 \mathrm{~mm}, r_{o}=2.5 \mathrm{~mm}$, and $p=10 \mathrm{~mm}$; (f) Effective refractive index comparison of the purely periodic and 4-fold twist-symmetric structures with dimensions: $h_{p}=1.2 \mathrm{~mm}$ (tuned: $h_{p}=1.075 \mathrm{~mm}$ ), $w_{p}=1 \mathrm{~mm}, r_{i}=1 \mathrm{~mm}, r_{o}=2.5 \mathrm{~mm}$, and $p=6 \mathrm{~mm}$.

produce a stop-band at selected frequencies between the first and second modes [13,17-19]. This stop-band is closed by applying glide symmetry, permitting the design of wideband flat metasurface lenses [20,21]. Moreover, while the stop-band between the first and second modes is suppressed in glide-symmetric structures, a wide stop-band can be created between the second and third modes. This stop-band can be used to design low-cost gap waveguides [22-24] and low-cost contact-less waveguide flanges for millimeter wave measurements [25]. Due to the beneficial properties of glide symmetry, several methods for fast calculation of the dispersion characteristics of glidesymmetric structures (Cartesian and polar) have been presented [8,26-28]. In these methods, by using the generalized Floquet theorem [4], the computational time is reduced and a valuable physical insight to the phenomenon of glide symmetry is provided [27].

Similar properties have been demonstrated in twistsymmetric structures [6-9]. Moreover, the possibility of reaching $m$-fold symmetry, where $m$ is any integer (in contrast to glide symmetry which is inherently of order 2 [4]), enables further control of the dispersion characteristics in twist-symmetric structures [6-9]. Such increased control has found applications in filters [7,8] and phase shifters [9], and has been proposed to be of use in fully metallic lowdispersive leaky-wave antenna designs [6-8]. This article provides an overview of the recent results in twist symmetries; three studies are conducted, simulated with the Eigenmode Solver of CST Microwave Studio [29], in which the properties of twist symmetry are highlighted. Firstly, in Section 2, a study of perfect twist symmetry is conducted and the refractive properties in structures possessing such symmetry are discussed. Secondly, in Section 3, polar glide symmetry is introduced and shown to provide further control of the propagation characteristics. Thirdly, in Section 4, the twist symmetry is broken, and the link between the symmetry and the opening and closing of stop-bands is illustrated. Finally, in Section 5, conclusions are drawn.

\section{Perfect twist symmetry}

Two different twist-symmetric configurations are considered: coaxial cables periodically loaded with either radially protruding square pins or coaxially aligned semi-circular holes. Both types of discontinuities (pins and holes) are placed on the inner conductor of the coaxial cable, and there is no electrical connection between the inner and outer conductors in any of the studied scenarios. Eight different configurations, i.e. purely periodic and 2-, 3- and 4 -fold twist-symmetric structures, for the two types of deformations, are studied and they are illustrated in Figures $1 \mathrm{a}-\mathrm{d}$ and $2 \mathrm{a}-\mathrm{d}$, for pin- and hole-type loading, respectively. The total periodicity, $p$, for all pin-loaded structures is $10 \mathrm{~mm}$. This means that the distance between two subsequent deformations is $10 \mathrm{~mm}$ in the purely periodic structure and $5 \mathrm{~mm}, 3.33 \mathrm{~mm}$ and $2.5 \mathrm{~mm}$ in the 2-, 3 - and 4-fold twist-symmetric structures, respectively. The radii of the inner, $r_{i}$, and outer, $r_{o}$, conductors are $1 \mathrm{~mm}$ and $2.5 \mathrm{~mm}$, respectively. The width of the square pins, $w_{p}$, is $1 \mathrm{~mm}$ and the pins are extruding with a height, $p_{h}$, of $1.2 \mathrm{~mm}$ from the inner conductor. Similarly, the total periodicity, $p$, for the holey structures is $10 \mathrm{~mm}$, resulting in similar deformation separation as in the pin-loaded structures. The radii of the inner conductor, $r_{i}$, is $2.3 \mathrm{~mm}$. The air gap, $g$, between the inner and outer conductor is $0.2 \mathrm{~mm}$. The holes are air-filled and have a semi-circular shape with an opening angle of $180^{\circ}$ and a radius of $r_{i}$. The holes have a length, $l_{h}$, of $2.2 \mathrm{~mm}$, extending along the coaxial transmission line.

The simulated dispersion diagrams for the four pin- and hole-loaded twist-symmetric structures are presented in Figures 1e and 2e, respectively. Similar behaviour is observed using both types of discontinuities. Notably, the stop-bands between the first $m$ modes are suppressed. This behaviour arises from the fact that the modes are eigenmodes of both the translational operation, applied to the full unit cell, and the twist symmetry operation, applied to the sub-unit cell, as is described by the 


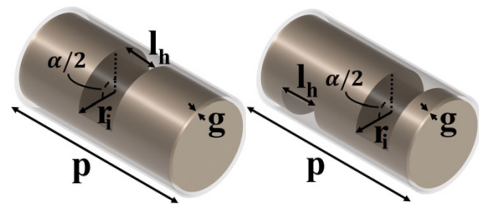

(a)

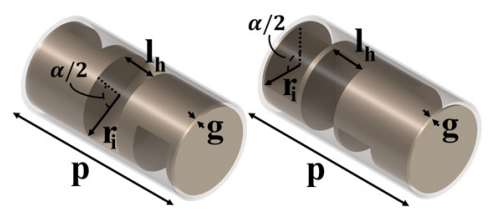

(c)

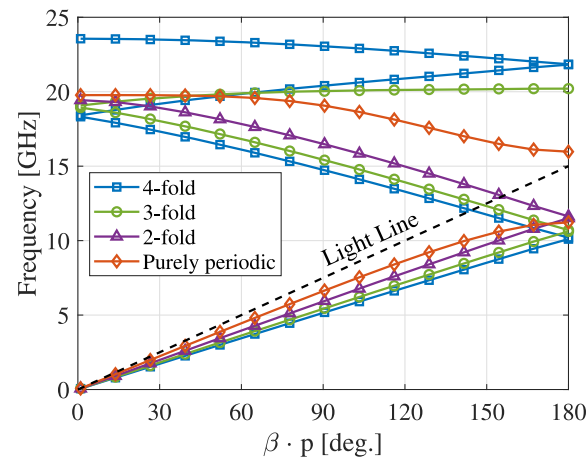

(e)

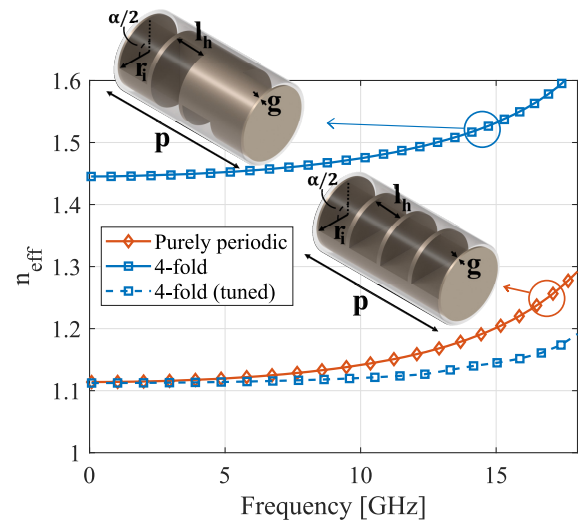

(f)

Fig. 2. Simulated perfect twist-symmetric structures with hole-type deformations: Studied structures: (a) purely periodic, (b) 2-fold, (c) 3-fold, and (d) 4-fold. (e) Dispersion diagram using the dimensions: $\alpha=180^{\circ}, l_{h}=2.2 \mathrm{~mm}, r_{i}=2.3 \mathrm{~mm}, g=0.2 \mathrm{~mm}$, and $p=10 \mathrm{~mm}$; (f) Effective refractive index comparison of the purely periodic and 4-fold twist-symmetric structures with dimensions: $\alpha=180^{\circ}$ (tuned: $\left.\alpha=85^{\circ}\right), l_{h}=2.2 \mathrm{~mm}, r_{i}=2.3 \mathrm{~mm}, g=0.2 \mathrm{~mm}$, and $p=10 \mathrm{~mm}$.

generalized Floquet theorem. More specifically, this theorem states that, in a twist-symmetric structure, the field is periodic after each twist operation, aside from a multiplication with an exponential factor $e^{j \beta p / m}[4]$. This leads to a degeneracy at the $m-1$ first edge points of the Brillouin diagram, and hence, there are no stop-bands at these points. Furthermore, only in the purely periodic structures does the first mode show significant dispersion. In all other cases, the suppression of stop-bands allows for a non-zero group velocity at the boundary, resulting in a less dispersive response. As the order of the symmetry is increased, an optically denser material is realized. This property can be used to produce broadband phase delays. For instance, in [9], a reconfigurable phase shifter, in which variable phase delays are achieved through reconfiguring the order of symmetry, has been demonstrated.

In order to provide a fair comparison between purely periodic structures and $m$-fold twist-symmetric structures $(m \geq 2)$, a purely periodic structure and a 4 -fold twistsymmetric structure, with the same distance between the deformations, are simulated. It means that the periodicity in the purely periodic structure is four times the periodicity in the 4-fold structure (see insets in Figs. 1f and 2f). Again, both pin- and hole-loading are considered. The total periodicity, $p$, in the pin-type structure is $6 \mathrm{~mm}$, and in the holey structure the periodicity is $10 \mathrm{~mm}$. All other dimensions are the same as in the previous study. Although, the true periodicity in the purely periodic structures is $p / 4$, for the sake of clear representation, 4 periods are employed in the simulations, so that the total length of the coaxial transmission lines is kept constant.

The effective refractive index of the the pin and holey structures are presented in Figures 1f and 2f, respectively. By applying twist symmetry to a periodic structure, increased density is obtained for structures with the same distance between two consecutive deformations. This is due to the fact that the wave is forced to propagate revolving around the twist axis rather than in a straight line, which leads to an increased phase delay per unit length [6]. Moreover, as the deformations may overlap in the longitudinal direction in twist-symmetric structures, which is not possible in purely periodic structures, even further increased density is possible. If the dimensions are tuned in the 4 -fold structures, so that the effective refractive index of the twist-symmetric and purely periodic structures matches in the long wavelength limit, it is clear that the frequency dispersion is lower in the twist-symmetric structures. This is illustrated with the dashed lines in Figures if and 2f. Here, we tune the pin height, $h_{p}$, to $1.075 \mathrm{~mm}$ and the opening angle of the hole, $\alpha$, to $85^{\circ}$ in the 4 -fold structures to obtain the same effective refractive index as in the purely periodic structures. In this example, the upper bound frequency, if $2 \%$ deviation from the effective refractive index in the long wavelength limit is allowed, increases from $8.8 \mathrm{GHz}$ to $11.1 \mathrm{GHz}$ in the pinloaded structure, and from $8.9 \mathrm{GHz}$ to $13.5 \mathrm{GHz}$ in the holeloaded structure.

\section{Polar glide symmetry}

Similar to Cartesian glide symmetry, the operations describing polar glide symmetry, which are applied to the sub-unit cell, are: a translation of $p / 2$ along the symmetry axis, followed by a mirroring. However, instead of mirroring the sub-unit cell with respect to a plane, the mirroring is done with respect to a cylindrical surface, coaxial with the periodicity axis and located at some radius, $R$.

To illustrate the properties of polar glide-symmetric structures, we analyze four coaxial transmission lines, loaded with coaxial half-cylindrical metallic extrusions, in which various degrees of symmetry are introduced. In such structures, the radius of the mirroring surface, $R$, is given by the geometrical mean radius of the two conductors of the coaxial cable, i.e. $R=\sqrt{r_{i} r_{o}}$. The four studied configurations are the: purely periodic, 2 -fold twist, perfect polar glide and 2-fold twisted polar glide structures, illustrated in Figure $3 \mathrm{a}-\mathrm{d}$, respectively. In the figures, the green metallic half-rings protrude from the inner conductor and red 


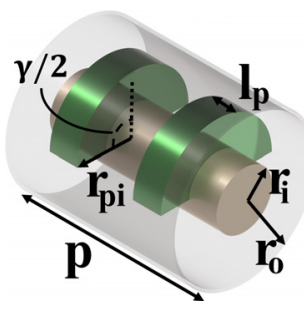

(a)

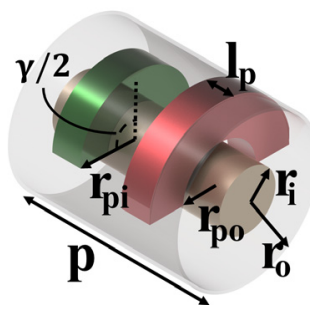

(c)

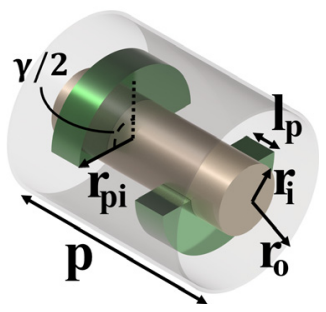

(b)

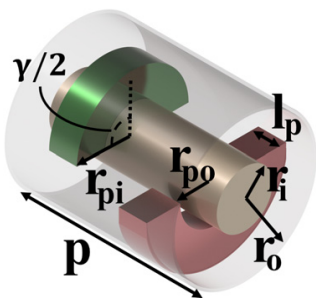

(d)

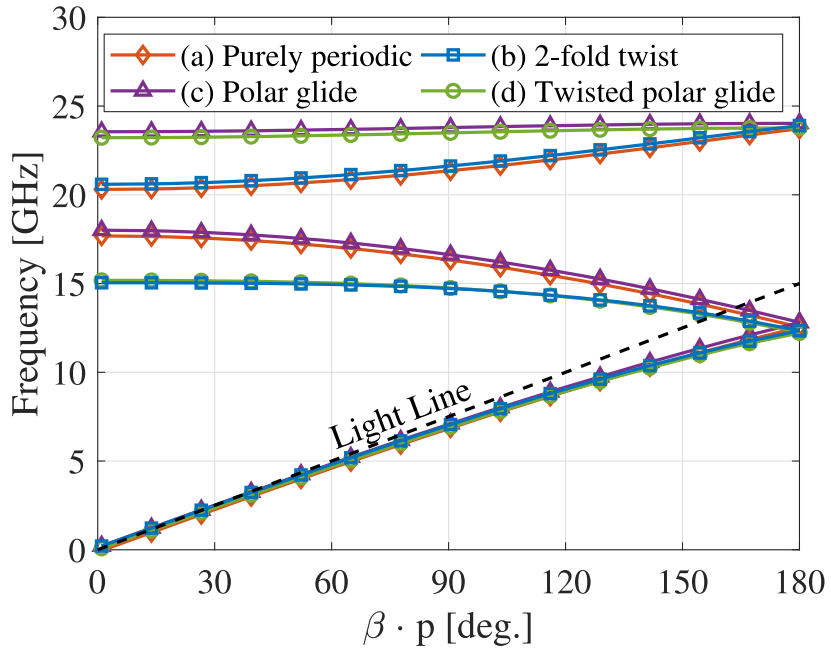

(e)

Fig. 3. Simulated structures in the study of polar glide symmetry. Studied structures: (a) purely periodic, (b) 2-fold twist, (c) polar glide, and (d) twisted polar glide symmetry; (e) Dispersion diagram using the dimensions: $\beta=180^{\circ}, r_{p i}=2.3 \mathrm{~mm}, r_{p o}=1.08 \mathrm{~mm}$, $l_{p}=1 \mathrm{~mm}, r_{i}=1 \mathrm{~mm}, r_{o}=2.5 \mathrm{~mm}$, and $p=6 \mathrm{~mm}$.

metallic half-rings protrude from the outer conductor of the coaxial cables, with no electrical connection between the two conductors. In the purely periodic structure (Fig. 3a), the minimal translational period is half of the period of the other configurations. The total periodicity, $p$, is $6 \mathrm{~mm}$ in all studied configurations. The radii of the inner, $r_{i}$, and outer, $r_{o}$, conductors are $1 \mathrm{~mm}$ and $2.5 \mathrm{~mm}$, respectively. The opening angle of the half-cylindrical pins, $\gamma$, is $180^{\circ}$, the longitudinal length of the half cylinders, $l_{p}$, is $1 \mathrm{~mm}$, the radius of the half cylinders attached to the inner conductor (in green), $r_{p i}$ is $2.3 \mathrm{~mm}$, and the radius to the half cylinders attached to the outer conductor (in red), $r_{p o}$, is $1.08 \mathrm{~mm}$ (obtained from performing the mirroring in the cylindrical surface at radius $R=\sqrt{r_{i} r_{o}}$ ).

The dispersion curves for all cases are presented in Figure 3e. The dispersion curve of the purely periodic structure is normalized with respect to $p$, which is the double of the minimal period of the structure, for coherence with the other cases. We first compare the periodic structure $3 \mathrm{a}$ (red line) with the polar glide one 3c (violet line), i.e. we study the effect of adding one of the two halfrings on the outer conductor instead of putting them both on the inner conductor. Similarly to 2 -fold twist symmetry, polar glide symmetry results in a closing of the stop-band at the first Brillouin zone boundary. For the first two modes, the effect of adding polar glide symmetry to a structure is small, compared to the purely periodic structure, i.e. the effect of adding a pin on the outer or inner conductor is the same. However, for the third mode, we see a significant upshift of the cut-off frequency in the polar glide structures, which can be used to widen stop-bands in between the second and third modes.

Now we discuss the 2 -fold twist in Figure 3 b, i.e. we study the effect of rotating one of the half-rings by $180^{\circ}$. In this case the optical density is higher, for the first two modes of propagation, compared to both the purely periodic and polar glide structures. However, the third mode is largely unaffected by the rotation of the second half-ring. This results in a widening of the stop-band between the second and third modes, but, in contrast to the effects of adding polar glide symmetry, this widening is caused by the downshift of the first and second mode, rather than the upshift of the third mode.

By combining 2-fold twist symmetry with polar glide, i.e. applying a translation, cylindrical mirroring and a $180^{\circ}$ rotation between each consecutive deformation, both the upshift of the third mode due the polar glide symmetry and the downshift of the first and second mode due to the 2-fold twist symmetry are obtained, and hence a large stop-band is opened in between the modes.

\section{Broken twist symmetry}

As explained in [4], an $m$-fold symmetry is required to close the stop-bands between the $m$ first modes. If this symmetry is broken, in one or several ways, stop-bands can be introduced in a controlled manner $[7,8]$. To illustrate this, we study a 4 -fold twist-symmetric structure, in which the symmetry is broken in two different ways.

The reference structure is a perfect 4-fold, as illustrated in Figure 4a. Similar to the structure discussed in Section 2, radially protruding pins, with square cross-sections, are attached to the inner conductor. These pins are denoted as 1,2,3 and 4 in the figure, and they are highlighted with the colours green, black, violet and red, respectively. In the initial structure, the total periodicity, $p$, is $10 \mathrm{~mm}$, the radii of the inner, $r_{i}$, and outer, $r_{o}$, conductors are $1 \mathrm{~mm}$ and $2.5 \mathrm{~mm}$, respectively, the width of the square pins, $w_{p}$, is $1 \mathrm{~mm}$ and the pins are extruding with a height, $p_{h}$, of $1.2 \mathrm{~mm}$ from the inner conductor. The dispersion curve, for the perfect 4-fold structure, is shown in blue in Figure 4d. Similar to the perfect 4-fold structures discussed above, there are no stop-bands between the first 4 modes. 


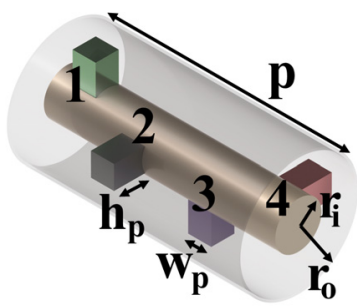

(a)

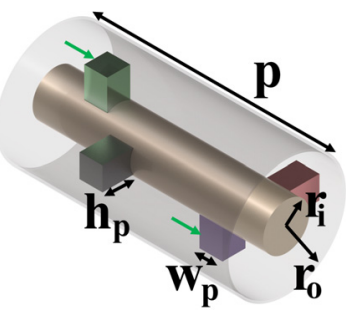

(b)

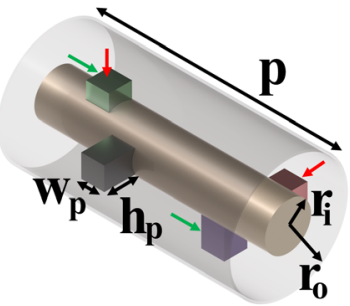

(c)

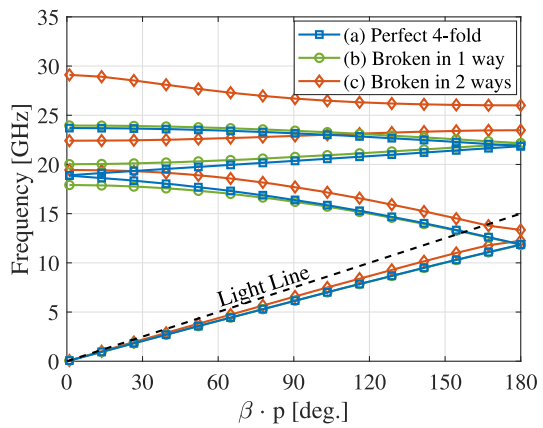

(d)

Fig. 4. Simulated structures for the observation of the symmetry breaking. Studied structures: (a) perfect 4-fold twist symmetry, (b) broken symmetry in 1 way, and (c) broken symmetry in two ways. (d) Dispersion diagram using the dimensions: $h_{p}=1.2$ mm, $w_{p}=1$ $\mathrm{mm}, r_{i}=1 \mathrm{~mm}, r_{o}=2.5 \mathrm{~mm}$, and $p=10 \mathrm{~mm}$. The first symmetry breaking is obtained by shifting pin 1 (green) and pin 3 (violet) by $1 \mathrm{~mm}$ according to green arrows. The second breaking is obtained by reducing heights of pin 1 (green) and 4 (red) by $0.6 \mathrm{~mm}$ according to red arrows.

A first breaking of the 4-fold symmetry is achieved by shifting pin 1 (green) and pin 3 (violet), $1 \mathrm{~mm}$ in the longitudinal direction, with respect to the other two pins, as is illustrated by the green arrows in Figure 4b. The resulting dispersion curve is shown in green in Figure 4d. Again, no stop-band is observed between modes 1 and 2, and modes 3 and 4 , since the structure still possesses 2 -fold twist symmetry. However, between modes 2 and 3, this structure exhibits a stop-band. The width of the stop-band can be tuned by varying the magnitude of the symmetry breaking $[7,8]$.

Next, the 2-fold symmetry of the structure in Figure $4 \mathrm{~b}$ is broken by reducing the height of pin 1 (green) and pin 4 (red) by $0.6 \mathrm{~mm}$, as illustrated by the red arrows in Figure 4c. This structure has no higher symmetry and, consequently, exhibits stop-bands in between all of the first 4 modes, as shown in the red curve in Figure 4d.

The breaking of symmetry, in combination with twisted polar glide symmetry, was used in [7] to design a fully metallic reconfigurable filter.

\section{Conclusions}

In this article, we provide an overview of the recent advances in the research regarding twist-symmetric structures. We illustrate the enhanced control of the dispersion properties, enabled by the twist symmetry operation, in periodically loaded coaxial transmission lines. More specifically, the reduced frequency dependence is highlighted and the impact of symmetry order on the phase constant is discussed. The direct relation between the higher symmetry and the absence of stop-bands between the $m$ first modes is demonstrated, together with an illustration of how full control of the pass and stop-bands can be obtained in twist-symmetric structures.

Additionally, it has been demonstrated that polar glide symmetry provides similar benefits as other types of higher symmetries (Cartesian glide and twist). In particular, polar glide is shown to create large stop-bands at high frequencies, and if combined with twist symmetries, the stop-band can be even further increased.
Twist symmetries find applications in fully metallic phase shifters and filters. Moreover, the reduced frequency dispersion may be employed in leaky-wave antennas with reduced beam-squint, appropriate for future millimeter wave communication networks and space applications [30].

\section{References}

1. P.J. Crepeau, P.R. McIsaac, Consequences of symmetry in periodic structures, Proc. IEEE 52, 33-43 (1963)

2. R. Mittra, S. Laxpati, Propagation in a wave guide with glide reflection symmetry, Can. J. Phys. 43, 353-372 (1965)

3. R. Kieburtz, J. Impagliazzo, Multimode propagation on radiating traveling-wave structures with glide-symmetric excitation, IEEE Trans. Antennas Propag. 18, 3-7 (1970)

4. A. Hessel, M.H. Chen, R.C.M. Li, A.A. Oliner, Propagation in periodically loaded waveguides with higher symmetries, Proc. IEEE 61, 183-195 (1973)

5. F. Ghasemifard, Periodic structures with higher symmetries: analysis and applications, Ph.D. dissertation, KTH Royal Institute of Technology, 2018

6. O. Dahlberg, R. Mitchell-Thomas, O. Quevedo-Teruel, Reducing the dispersion of periodic structures with twist and polar glide symmetries, Sci. Rep. 7, 10136 (2017)

7. F. Ghasemifard, M. Norgren, O. Quevedo-Teruel, Twist and polar glide symmetries: an additional degree of freedom to control the propagation characteristics of periodic structures, Sci. Rep. 8, 11266 (2018)

8. Q. Chen, F. Ghasemifard, G. Valerio, O. Quevedo-Teruel, Modeling and dispersion analysis of coaxial lines with higher symmetries, IEEE Trans. Microw. Theory Tech. 66, 1-8 (2018)

9. O. Quevedo-Teruel, O. Dahlberg, G. Valerio, Propagation in waveguides with transversal twist-symmetric holey metallic plates, IEEE Microw. Wirel. Compon. Lett. 28, 1-3, (2018)

10. W. Cao, Z.N. Chen, W. Hong, B. Zhang, A. Liu, A beam scanning leaky-wave slot antenna with enhanced scanning angle range and flat gain characteristic using composite phase-shifting transmission line, IEEE Trans. Antennas Propag. 62, 5871-5875 (2014) 
11. J.J. Wu, C.-J. Wu, D.J. Hou, K. Liu, T.J. Yang, Propagation of low-frequency spoof surface plasmon polaritons in a bilateral cross-metal diaphragm channel waveguide in the absence of bandgap, IEEE Photonics J. 7, 1-8 (2015)

12. Y.-L. Lyu, X.-X. Liu, P.-Y. Wang, D. Erni, Q. Wu, C. Wang, N.-Y. Kim, F.-Y. Meng, Leaky-wave antennas based on noncutoff substrate integrated waveguide supporting beam scanning from backward to forward, IEEE Trans. Antennas Propag. 64, 2155-2164 (2016)

13. R. Quesada, D. Martín-Cano, F. García-Vidal, J. BravoAbad, Deep-subwavelength negative-index waveguiding enabled by coupled conformal surface plasmons, Opt. Lett. 39, 2990-2993 (2014)

14. D. Cavallo, C. Felita, Analytical formulas for artificial dielectrics with nonaligned layers, IEEE Trans. Antennas Propag. 65, 5303-5311 (2017)

15. D. Cavallo, Dissipation losses in artificial dielectric layers, IEEE Trans. Antennas Propag. 66, 7460-7465 (2018)

16. T. Chang, J.U. Kim, S.K. Kang, H. Kim, D.K. Kim, Y.-H. Lee, J. Shin, Broadband giant-refractive-index material based on mesoscopic space-filling curves, Nat. Commun. 7, 12661 (2016)

17. P. Padilla, L. Herrán, A. Tamayo-Domínguez, J. ValenzuelaValdés, O. Quevedo-Teruel, Glide symmetry to prevent the lowest stopband of printed corrugated transmission lines, IEEE Microw. Wirel. Compon. Lett. 28, 1-3 (2018)

18. M. Camacho, R.C. Mitchell-Thomas, A.P. Hibbins, J.R. Sambles, O. Quevedo-Teruel, Designer surface plasmon dispersion on a one-dimensional periodic slot metasurface with glide symmetry, Opt. Lett. 42, 3375-3378 (2017)

19. M. Camacho, R.C. Mitchell-Thomas, A.P. Hibbins, J.R. Sambles, O. Quevedo-Teruel, Mimicking glide symmetry dispersion with coupled slot metasurfaces, Appl. Phys. Lett. 111, 121603 (2017)

20. O. Quevedo-Teruel, M. Ebrahimpouri, M.N.M. Kehn, Ultrawideband metasurface lenses based on off-shifted opposite layers, IEEE Antennas Wirel. Propag. Lett. 15, 484-487 (2016)
21. O. Quevedo-Teruel, J. Miao, M. Mattsson, A. AlgabaBrazalez, M. Johansson, L. Manholm, Glide-symmetric fully metallic Luneburg lens for $5 \mathrm{G}$ communications at Ka-band, IEEE Antennas Wirel. Propag. Lett. 17, 15881592 (2018)

22. M. Ebrahimpouri, O. Quevedo-Teruel, E. Rajo-Iglesias, Design guidelines for gap waveguide technology based on glide-symmetric holey structures, IEEE Microw. Wirel. Compon. Lett. 27, 542-544 (2017)

23. M. Ebrahimpouri, E. Rajo-Iglesias, Z. Sipus, O. QuevedoTeruel, Cost-effective gap waveguide technology based on glide-symmetric holey EBG structures, IEEE Trans. Microw. Theory Tech. 66, 927-934 (2018)

24. E. Rajo-Iglesias, M. Ebrahimpouri, O. Quevedo-Teruel, Wideband phase shifter in groove gap waveguide technology implemented with glide-symmetric holey EBG, IEEE Microw. Wirel. Compon. Lett. 28, 476-478 (2018)

25. M. Ebrahimpouri, A.A. Brazalez, L. Manholm, O. QuevedoTeruel, Using glide-symmetric holes to reduce leakage between waveguide flanges, IEEE Microw. Wirel. Compon. Lett. 28, 473-475 (2018)

26. G. Valerio, Z. Sipus, A. Grbic, O. Quevedo-Teruel, Accurate equivalent-circuit descriptions of thin glide-symmetric corrugated metasurfaces, IEEE Trans. Antennas Propag. 65, 2695-2700 (2017)

27. G. Valerio, F. Ghasemifard, Z. Sipus, O. Quevedo-Teruel, Glide-symmetric all-metal holey metasurfaces for lowdispersive artificial materials: modeling and properties, IEEE Trans. Microw. Theory Tech. 66, 1-14 (2018)

28. F. Ghasemifard, M. Norgren, O. Quevedo-Teruel, Dispersion analysis of 2-D glide-symmetric corrugated metasurfaces using mode-matching technique, IEEE Microw. Wirel. Compon. Lett. 28, 1-3 (2018)

29. CST Microwave Studio, Version, 2017. http://www.cst. com/ Accessed November 21, 2018

30. O. Quevedo-Teruel, M. Ebrahimpouri, F. Ghasemifard, Lens antennas for $5 \mathrm{G}$ communications systems, IEEE Commun. Mag. 56, 36-41 (2018)

Cite this article as: Oskar Dahlberg, Fatemeh Ghasemifard, Guido Valerio, Oscar Quevedo-Teruel, Propagation characteristics of periodic structures possessing twist and polar glide symmetries, EPJ Appl. Metamat. 6, 14 (2019) 